\title{
AN EXTENSION OF OSGOOD'S OSCILLATION THEOREM FOR A NONLINEAR DIFFERENTIAL EQUATION
}

\author{
CHOY-TAK TAAM
}

1. Introduction. After examining critically a theorem of Bôcher, Osgood proved the following oscillation theorem [1]:

TheOREM 1. Let

(a) $f(t)$ be continuous and monotonic in the interval $T \leqq t<\infty$,

(b) $f(t)>f_{0}>0$ in $T \leqq t<\infty, f_{0}$ being a constant.

Then an arbitrary solution of the differential equation

$$
x^{\prime \prime}+f(t) x=0
$$

oscillates an infinite number of times in any interval $T \leqq T^{\prime} \leqq t<\infty$ in which the amplitudes of the oscillations are uniformly bounded. Moreover, the amplitudes vary monotonically, increasing when $f(t)$ decreases monotonically, and decreasing in the opposite case.

If, furthermore, $f(t)$ is uniformly bounded, the amplitudes of the oscillations do not become less than a certain positive constant.

Under the conditions stated above (including the uniform bound on $f(t)$ ), clearly $f(t)$ tends to a positive limit $a^{2}$, say, as $t \rightarrow \infty$. If $f(t)$ is so "near" to $a^{2}$ (but not necessarily tends to a limit) such that the integral

$$
\int_{T}^{\infty}\left|f(t)-a^{2}\right| d t
$$

exists, it is known that to each solution $x(t)$ of (1.1) there exists a solution $A \sin (a t+B)$ of

$$
x^{\prime \prime}+a^{2} x=0
$$

such that

$$
x(t)=A \sin (a t+B)+\epsilon(t),
$$

where $\epsilon(t)$ tends to zero as $t$ tends to infinity [2]. Whether (1.3) holds or not under the conditions of Osgood's theorem is not clear to the author. However we can exhibit another property of (1.1) which resembles the equation (1.2), namely the trajectory of a solution $x(t)$ of $(1.1)$ is

Presented to the Society, December 30, 1953; received by the editors October 5, 1953 and, in revised form, February 15, 1954. 


$$
x^{\prime 2}+a^{2} x^{2}=c^{2}+\epsilon(t)
$$

for some $c^{2}>0$, where $\epsilon(t)$ tends to zero as $t$ tends to infinity. To prove this, multiplying (1.1) by $2 x^{\prime}$ and integrating the result from $T$ to $t$, we obtain

$$
{x^{\prime}}^{2}+f x^{2}=c_{1}^{2}+\int_{T}^{t} x^{2} d f, \quad c_{1}^{2}>0,
$$

the integral being a Stieltjes integral. Since $x(t)$ is bounded and $f(t)$ tends monotonically to a limit $a^{2}$, the integral in (1.5) converges to a limit as $t$ tends to infinity. Rewriting (1.5)

$$
x^{\prime 2}+a^{2} x^{2}=c^{2}+\left(a^{2}-f\right) x^{2}-\int_{t}^{\infty} x^{2} d f
$$

we have the result (1.4). We note that (1.4) also shows that the amplitudes of the oscillations and the velocities at the center of the oscillation tend to limits as $t$ tends to infinity.

Butlewski [3] extended Osgood's result to the self-adjoint differential equation $\left(m(t) x^{\prime}\right)^{\prime}+f(t) x=0$. In this paper we shall extend further the result to a nonlinear differential equation (2.1).

2. Extension of Osgood's theorem. Consider the nonlinear differential equation

$$
\left(m(t) x^{\prime}\right)^{\prime}+\sum_{i=1}^{b} f_{i}(t) x^{2 i-1}=0,
$$

where $(m(t))^{-1}, f_{1}(t), f_{2}(t), \cdots$, and $f_{k}(t)$ are real-valued, non-negative, Lebesgue-measurable functions in the interval $T \leqq t<\infty$, and Lebesgue-integrable in $T \leqq t \leqq R$ for every $R>T$. By a solution of (2.1) we mean a function $x(t)$ which is absolutely continuous and $m(t) x^{\prime}(t)$ is equal almost everywhere to an absolutely continuous function, say $M(t)$, such that (2.1) is satisfied almost everywhere if $m(t) x^{\prime}(t)$ is replaced by $M(t)$. We shall consider only real-valued, nontrivial solutions.

We shall prove the following extension of Osgood's theorem.

TheOREM 2. Suppose that for $T \leqq t<\infty$

(a) $m(t)<m_{0}, m_{0}$ being a positive constant,

(b) $f_{1}(t)>f_{0}, f_{0}$ being a positive constant,

(c) $m(t) f_{i}(t) \geqq 0, i=1,2, \cdots, k$,

(d) $m(t) f_{i}(t) \geqq r>0$ for some $i, r$ being a constant, 
(e) all $m(t) f_{i}(t)$ are nondecreasing or all are nonincreasing, $i$ $=1,2, \cdots, k$.

Then any solution of the equation (2.1) oscillates an infinite number of times in any interval $T \leqq T^{\prime}<t<\infty$ in which the amplitudes of the oscillations are uniformly bounded. Moreover, the amplitudes vary monotonically, increasing when all $m(t) f_{i}(t)$ decrease monotonically, and decreasing in the opposite case.

If, furthermore, all $m(t) f_{i}(t)$ are uniformly bounded, the amplitudes of the oscillations do not become less than a certain positive constant.

Proof. First we show that any solution of (2.1) oscillates an infinite number of times in any interval $T \leqq T^{\prime} \leqq t<\infty$. To do this, we compare (2.1) with

$$
m_{0} y^{\prime \prime}+f_{0} y=0 .
$$

Every solution of (2.2) oscillates an infinite number of times in any interval $T \leqq T^{\prime} \leqq t<\infty$. Let $t_{1}$ and $t_{2}$ be any consecutive zeros of a solution $y(t)$ of (2.2). We shall prove that every solution $x(t)$ of $(2.1)$ vanishes at least once in $t_{1} \leqq t \leqq t_{2}$. Suppose that this is not true; then $x(t)$ does not vanish on $t_{1} \leqq t \leqq t_{2}$. It is easy to verify that (the Picone formula, see $[6$, p. 226])

$$
\begin{aligned}
\frac{d}{d t}\left[\frac{y}{x}\left(m_{0} x y^{\prime}-m x^{\prime} y\right)\right] \\
=\left(\sum_{i=1}^{k} f_{i} x^{2(i-1)}-f_{0}\right) y^{2}+\left(m_{0}-m\right) y^{\prime 2}+m \frac{\left(x y^{\prime}-x^{\prime} y\right)^{2}}{x^{2}}
\end{aligned}
$$

holds almost everywhere, and integrating from $t_{1}$ to $t_{2}$ we have

$$
\begin{aligned}
{\left[\frac{y}{x}\left(m_{0} x y^{\prime}-m x^{\prime} y\right)\right]_{t_{1}}^{t_{2}} } & \\
=\int_{t_{1}}^{t_{2}}\left[\left(\sum_{i=1}^{k} f_{i} x^{2(i-1)}-f_{0}\right) y^{2}\right. & +\left(m_{0}-m\right) y^{\prime 2} \\
& \left.+m \frac{\left(x y^{\prime}-x^{\prime} y\right)^{2}}{x^{2}}\right] d t .
\end{aligned}
$$

The equation (2.3) leads to contradiction, because the integral is definitely positive while the left-hand member is zero. This proves that every solution $x(t)$ of (2.1) vanishes at least once in $t_{1} \leqq t \leqq t_{2}$ and so oscillates an infinite number of times in $T \leqq T^{\prime} \leqq t<\infty$. (Using a known method with some modification [6, p. 226], $x(t)$ can be shown 
to vanish at least once in $t_{1}<t<t_{2}$.)

Let $M(t)$ be the absolutely continuous function to which $m(t) x^{\prime}(t)$ is equal almost everywhere. $M(t)$ is called the momentum. We note that $M(t)$ does not vanish identically in any interval $I$; otherwise, from (2.1), $x(t)$ must also vanish identically in $I$ and so $x(t)=0$. Since

$$
\begin{gathered}
x\left(t_{2}\right)-x\left(t_{1}\right)=\int_{t_{1}}^{t_{2}} \frac{M(t)}{m(t)} d t, \\
M\left(t_{1}\right)-M\left(t_{2}\right)=\sum_{i=1}^{k} \int_{t_{1}}^{t_{2}} f_{i} x^{2 i-1} d t,
\end{gathered}
$$

it is clear that between any consecutive zeros of $x(t)$ there is at least one zero of $M(t)$, and between any consecutive zeros of $M(t)$ there is at least one zero of $x(t)$. It follows that between any consecutive zeros of $x(t)(M(t))$ there is one and only one zero of $M(t)(x(t))$. It is also clear that $x(t)(M(t))$ assumes its maxima and minima at the zeros of $M(t)(x(t))$.

Denote the zeros of $x(t)$ by $t_{2 n}$, of $M(t)$ by $t_{2 n+1}, n=0,1,2, \cdots$, and $t_{i}<t_{j}$ for $i<j$. Set

$$
X_{2 n+1}=\left|x\left(t_{2 n+1}\right)\right|, \quad M_{2 n}=\left|M\left(t_{2 n}\right)\right|, \quad F_{i, n}=m\left(t_{n}\right) f_{i}\left(t_{n}\right),
$$

for $n=0,1,2, \cdots$.

Multiplying (2.1) by $M=m x^{\prime}$, we get

$$
M M^{\prime}+\sum_{i=1}^{k} m f_{i} x^{\prime} x^{2 i-1}=0
$$

almost everywhere. Integrating (2.4) from $t_{2 n}$ to $t_{2 n+1}$, we have

$$
\frac{1}{2} M_{2 n}^{2}=\sum_{i=1}^{k} \int_{t_{2 n}}^{t_{2 n+1}} m f_{i} x^{\prime} x^{2 i-1} d t .
$$

Similarly integration of (2.4) from $t_{2 n+1}$ to $t_{2 n+2}$ gives

$$
\frac{1}{2} M_{2 n+2}^{2}=-\sum_{i=1}^{k} \int_{t_{2 n+1}}^{t_{2 n+2}} m f_{i} x^{\prime} x^{2 i-1} d t .
$$

We observe that from $t_{2 n}$ to $t_{2 n+1}, x^{\prime}$ and $x$ have the same sign, from $t_{2 n+1}$ to $t_{2 n+2}$ opposite signs.

Case I. $m(t) f_{i}(t)$ nonincreasing for all $i$ and $m(t) f_{i}(t) \geqq r>0$ for some $i$. From (2.5), clearly

$$
M_{2 n}^{2} \geqq \sum_{i=1}^{k} F_{i, 2 n+1} \frac{X_{2 n+1}^{2 i}}{i} .
$$


And from (2.6)

$$
M_{2 n+2}^{2} \leqq \sum_{i=1}^{k} F_{i, 2 n+1} \frac{X_{2 n+1}^{2 i}}{i} .
$$

It follows that the momenta at the zeros of $x(t)$

$$
M_{0}, M_{2}, M_{4}, \cdots, M_{2 n}, \cdots
$$

form a nonincreasing sequence. Since $m(t) f_{i}(t) \geqq r>0$ for some $i=j$, say, from $(2.7)$, then

$$
M_{0}^{2} \geqq M_{2 n}^{2} \geqq F_{j, 2 n+1} \frac{X_{2 n+1}^{2 j}}{j} \geqq \frac{r}{j} X_{2 n+1}^{2 j} .
$$

This proves that $X_{2 n+1}$ is bounded for all $n$. From (2.6), replacing $n$ by $n-1$, we have

$$
\frac{1}{2} M_{2 n}^{2}=-\sum_{i=1}^{k} \int_{t_{2 n-1}}^{t_{2 n}} m f_{i} x^{\prime} x^{2 i-1} d t
$$

and so

$$
M_{2 n}^{2} \geqq \sum_{i=1}^{k} F_{i, 2 n} \frac{X_{2 n-1}^{2 i}}{i} .
$$

From (2.5), we have

$$
M_{2 n}^{2} \leqq \sum_{i=1}^{k} F_{i, 2 n} \frac{X_{2 n+1}^{2 i}}{i} .
$$

(2.12) and (2.13) then yield

$$
\sum_{i=1}^{k} F_{i, 2 n} \frac{X_{2 n-1}^{2 i}}{i} \leqq \sum_{i=1}^{b} F_{i, 2 n} \frac{X_{2 n+1}^{2 i}}{i}
$$

which implies $X_{2 n-1} \leqq X_{2 n+1}$ and so the amplitudes of the oscillations

$$
X_{1}, X_{3}, X_{5}, \cdots, X_{2 n+1}, \cdots
$$

form a nondecreasing sequence and converge to a positive limit. From (2.10) we have

$$
M_{2 n}^{2} \geqq \frac{r}{j} X_{2 n+1}^{2 j} \geqq \frac{r}{j} X_{1}^{2 j}>0 .
$$

It follows that the sequence (2.9) of momenta also converges to a positive limit. 
Case II. $m(t) f_{i}(t)$ nondecreasing and remaining finite for all $i$. From (2.5), we obtain

$$
M_{2 n}^{2} \geqq \sum_{i=1}^{k} F_{i, 2 n} \frac{X_{2 n+1}^{2 i}}{i},
$$

and from (2.11)

$$
M_{2 n}^{2} \leqq \sum_{i=1}^{k} F_{i, 2 n} \frac{X_{2 n-1}^{2 i}}{i} .
$$

(2.15) and (2.16) then give

$$
\sum_{i=1}^{b} F_{i, 2 n} \frac{X_{2 n+1}^{2 i}}{i} \leqq \sum_{i=1}^{k} F_{i, 2 n} \frac{X_{2 n-1}^{2 i}}{i}
$$

which implies that $X_{2 n+1} \leqq X_{2 n-1}$ and so the amplitudes

$$
X_{1}, X_{3}, X_{5}, \cdots, X_{2 n+1}, \cdots
$$

form a nonincreasing sequence. From (2.5), we have also

$$
M_{2 n}^{2} \leqq \sum_{i=1}^{b} F_{i, 2 n+1} \frac{X_{2 n+1}^{2 i}}{i},
$$

and from (2.6)

$$
M_{2 n+2}^{2} \geqq \sum_{i=1}^{k} F_{i, 2 n+1} \frac{X_{2 n+1}^{2 i}}{i} .
$$

Since (2.19) and (2.20) imply that $M_{2 n+2} \geqq M_{2 n}$, the sequence of momenta

$$
M_{0}, M_{2}, M_{4}, \cdots, M_{2 n}, \cdots
$$

is nondecreasing. If $B$ is an upper bound of $m(t) f_{i}(t)$ for all $i$, then, from (2.19),

$$
M_{2 n}^{2} \leqq B \sum_{i=1}^{k} \frac{X_{2 n+1}^{2 i}}{i}
$$

and so

$$
\sum_{i=1}^{k} \frac{X_{2 n+1}^{2 i}}{i} \geqq \frac{M_{2 n}^{2}}{B} \geqq \frac{M_{0}^{2}}{B}>0 .
$$

Since (2.23) implies that $X_{2 n+1}$ is bounded below, the nonincreasing 
sequence (2.18) converges to a positive limit. Then, from (2.22), $M_{2 n}$ is bounded above. It follows that the nondecreasing sequence (2.21) also converges to a limit.

This completes the proof of Theorem 2.

In the following corollary, which is similar to (1.4), let the conditions a to e of Theorem 2 be satisfied and let all $F_{i}(t)=m(t) f_{i}(t)$ be uniformly bounded. Then each $F_{i}(t)$ tends to some non-negative limit $a_{i}$, say, as $t$ tends to infinity.

Corollary 2.1. The trajectory of a solution $x(t)$ of $(2.1)$ is

$$
M^{2}+\sum_{i=1}^{k} a_{i} \frac{x^{2 i}}{i}=c^{2}+\epsilon(t)
$$

for some constant $c \neq 0$, where $\epsilon(t)$ tends to zero as $t$ tends to infinity.

Proof. Integrating (2.4) from $T$ to $t$, we obtain

$$
\left[M^{2}\right]_{T}^{t}+2 \sum_{i=1}^{k} \int_{T}^{t} F_{i} x^{\prime} x^{2 i-1} d t=0
$$

and so (integration by parts (see $[4$, p. 332]))

$$
M^{2}+\sum_{i=1}^{k} F_{i} \frac{x^{2 i}}{i}=c_{1}^{2}+\sum_{i=1}^{k} \frac{1}{i} \int_{T}^{t} x^{2 i} d F_{i} \text {. }
$$

Since $x(t)$ is bounded, each integral in (2.26) converges to a limit as $t$ tends to infinity. Splitting the integrals, (2.26) can be written

$$
M^{2}+\sum_{i=1}^{k} a_{i} \frac{x^{2 i}}{i}=c^{2}+\epsilon(t)
$$

for some constant $c \neq 0$, where

$$
\epsilon(t)=\sum_{i=1}^{k}\left(a_{i}-F_{i}\right) \frac{x^{2 i}}{i}-\sum_{i=1}^{k} \frac{1}{i} \int_{t}^{\infty} x^{2 i} d F_{i} .
$$

Clearly $\epsilon(t)$ tends to zero as $t$ tends to infinity.

3. Sufficient conditions for the boundedness of solutions. The following theorem gives another set of conditions for the boundedness of the solutions of (2.1).

Theorem 3. Suppose that, for $T \leqq t<\infty$, either a, b, c or a, b, d of the following conditions are satisfied:

(a) $m(t) f_{i}(t) \geqq 0, i=1,2,3, \cdots, k$,

(b) $m(t) f_{i}(t)$ is monotonic, $i=1,2,3, \cdots, k$, 
(c) $m(t) f_{i}(t)$ is nondecreasing and positive for some $i$,

(d) $m(t) f_{i}(t)$ is nonincreasing for all $i$ and has a positive lower bound for some $i$.

Then every solution of (2.1) is bounded on $T \leqq t<\infty$.

The method of $\$ 2$ does not apply here because under the conditions of Theorem 3 the solutions may not oscillate. For example

$$
\left(t^{2} x^{\prime}\right)^{\prime}+\frac{1}{t^{2}} x=0
$$

has the general solution

$$
x(t)=A \sin \left(\frac{1}{t}+B\right)
$$

which is bounded and does not oscillate for large $t$.

Proof of Theorem 3. Set $F_{i}=m f_{i}$. Integrating (2.4) from $T$ to $t$ and evaluating the result by integration by parts, we have

$$
M^{2}+\sum_{i=1}^{k} F_{i} \frac{x^{2 i}}{i}=c^{2}+\sum_{i=1}^{k} \frac{1}{i} \int_{T}^{t} x^{2 i} d F_{i} .
$$

Case I. $F_{i}$ is nonincreasing for all $i$ and for some $i=j$, say, $F_{i}$ has a positive lower bound. Then each integral in (3.1) is nonpositive, and we have

$$
F_{j} \frac{x^{2 j}}{j} \leqq c^{2}
$$

which implies that $x(t)$ is bounded. Clearly $M$ is also bounded.

Case II. $F_{i}$ is nondecreasing and positive for some $i$. Let the nondecreasing $F_{i}$ be $F_{i(p)}, p=1,2, \cdots, h$. Then from (3.1) we have

$$
\sum_{p=1}^{h} F_{i(p)} \frac{x^{2 i(p)}}{i(p)} \leqq c^{2}+\sum_{p=1}^{h} \frac{1}{i(p)} \int_{T}^{t} x^{2 i(p)} d F_{i(p)} .
$$

Suppose that the maximum $H$ of $|x(t)|$ on $[T, t]$ is assumed at $t=t_{0}$. Then

$$
\sum_{p=1}^{h} F_{i(p)}\left(t_{0}\right) \frac{H^{2 i(p)}}{i(p)} \leqq c^{2}+\sum_{p=1}^{h} \frac{1}{i(p)} \int_{T}^{t_{0}} x^{2 i(p)} d F_{i(p)}
$$

and

$$
\sum_{p=1}^{h} F_{i(p)}\left(t_{0}\right) \frac{H^{2 i(p)}}{i(p)} \leqq c^{2}+\sum_{p=1}^{h} \frac{H^{2 i(p)}}{i(p)} \int_{T}^{t_{0}} d F_{i(p) .}
$$


It follows that

$$
\sum_{p=1}^{h} F_{i(p)}(T) \frac{H^{2 i(p)}}{i(p)} \leqq c^{2}
$$

If $F_{i(p)}(T)$ is positive for $p=g$, then

$$
F_{i(g)}(T) \frac{H^{2 i(g)}}{i(g)} \leqq c^{2}
$$

which implies that $H$ is bounded. This completes the proof.

Theorem 3 is a generalization of a theorem of W. Leighton [5].

4. A question of existence and uniqueness of solutions. In $\$ \S 2$ and 3 we assume the existence and uniqueness of the solutions of (2.1) on $T \leqq t<\infty$. Since the standard existence theorems which can be applied to equation (2.1) are local in nature, we give here a brief proof of our assumption. Clearly it is sufficient to prove the existence and uniqueness of the solutions on $[T, t]$ for every $t>T$ under the conditions stated in the first paragraph of $\$ 2$ and in Theorem 3. Write equation (2.1) as a system of equations

$$
x^{\prime}=\frac{1}{m(t)} M, \quad M^{\prime}=-\sum_{i=1}^{k} f_{i}(t) x^{2 i-1} .
$$

Let $x_{0}$ and $M_{0}$ be any two constants. Imagine that there is a set of solutions $x(t)$ and $M(t)$ satisfying the conditions $x(T)=x_{0}, M(T)$ $=M_{0}$ and determine an upper bound $B_{1}$ for $x(t)$ and an upper bound $B_{2}$ for $M(t)$ on $[T, t] . B_{1}$ may be determined by (3.2) or (3.7) and $B_{2}$ (using the notations of $\S 3$ and (3.1)) by $M^{2} \leqq c^{2}$ or

$$
M^{2} \leqq c^{2}+\sum_{p=1}^{h} \frac{B_{1}^{2 i(p)}}{i(p)} \int_{T}^{t} d F_{i(p)} .
$$

Clearly $B_{1}$ and $B_{2}$ depend on $x_{0}, M_{0}$ and $F_{i}$ only. Let $K(t)$ be a function of class $L(T, t)$ such that

$$
\sum_{i=1}^{k} f_{i}(t)\left|x_{2}^{2 i-1}-x_{1}^{2 i-1}\right| \leqq K(t)\left|x_{2}-x_{1}\right|
$$

whenever $\left|x_{2}\right| \leqq 2 B_{1},\left|x_{1}\right| \leqq 2 B_{1}$. Determine a positive constant $h$ such that

$$
B_{2} \int_{t_{1}}^{t_{2}} \frac{d t}{m(t)}<\frac{B_{1}}{2}, \quad B_{1} \int_{t_{1}}^{t_{2}} K(t) d t<\frac{B_{2}}{2}
$$


whenever $0 \leqq t_{2}-t_{1} \leqq h, t_{1}$ and $t_{2}$ being points in $[T, t]$. Then by the method of successive approximations we can prove that there exists a set of solutions $x(t)$ and $M(t)$ satisfying (4.1) almost everywhere at least in $[T, T+h]$ and $x(T)=x_{0}, M(t)=M_{0}$; moreover the solutions are unique. Since $|x(t)|$ and $|M(t)|$ are bounded by $B_{1}$ and $B_{2}$ respectively (see the proof of Theorem 3 ) in $\left[T, T_{1}\right], T_{1}=T+h$, by the method of successive approximations again, there exists a set of solutions $x_{1}(t)$ and $M_{1}(t)$ satisfying (4.1) almost everywhere at least in $\left[T, T_{2}\right], T_{2}=T+2 h$, and $x_{1}\left(T_{1}\right)=x\left(T_{1}\right), M_{1}\left(T_{1}\right)=M\left(T_{1}\right)$; moreover the two sets of solutions are equal at the points of the common part of their intervals of existence. Repeating this process of continuation a finite number of times, we see that there exists a unique set of solutions $x(t)$ and $M(t)$ satisfying (4.1) almost everywhere in $[T, t]$ and $x(T)=x_{0}, M(T)=M_{0}$.

5. Further extensions. Let all the differential equations discussed in this section admit of unique solutions on $T \leqq t<\infty$. Let $f\left(t, x, x^{\prime}\right)$ be a function such that

$$
x^{\prime} f\left(t, x, x^{\prime}\right) \geqq 0
$$

and $m(t), f_{i}(t)$ satisfy the conditions stated in the first paragraph of $\S 2$. We observe that Theorem 3 still holds for the equation

$$
\left(m x^{\prime}\right)^{\prime}+f+\sum_{i=1}^{k} f_{i} x^{2 i-1}=0 .
$$

In fact Theorem 3 remains true even for these more general differential equations of first order and of second order

$$
\begin{gathered}
\left(m x^{\prime}\right)^{\prime}+f+\sum_{i=1}^{k} f_{i} g_{i}=0, \\
m f+\sum_{i=1}^{k} m f_{i} g_{i}=0,
\end{gathered}
$$

where $g_{i}=g_{i}(x)$ is a continuous function of $x, g_{i}(x)=-g_{i}(-x) \geqq 0$ for $x \geqq 0$, provided there is an $i$ such that when $m f_{i}$ has a positive lower bound the integral

$$
\int_{0}^{x} g_{i}(x) d x
$$

is divergent as $x \rightarrow \infty$.

We observe that Theorem 2 holds also for the differential equation 


$$
\left(m x^{\prime}\right)^{\prime}+\sum_{i=1}^{k} f_{i}(t) x^{n(i)} u_{i}(x)=0
$$

provided that the following conditions are satisfied:

(a) $n(1)=1, n(i)=p(i) / q(i) \geqq 1$ for $i>1$, where $p(i)$ and $q(i)$ are positive odd integers,

(b) $u_{1}(x)=1, u_{i}(x)=u_{i}(-x)>0$ for $x>0$, and $u_{i}(x)$ is continuous for all $x$,

(c) there is an $i$ such that when $m f_{i}$ has a positive lower bound the integral

$$
\int_{0}^{x} x^{n(i)} u_{i}(x) d x
$$

is divergent as $x \rightarrow \infty$.

All the remarks in this section can be proved by the method of $\$ 2$ or $\S 3$. To prove that Theorem 3 holds for the equation (5.3), what one needs is to replace (3.1) by

$$
\begin{aligned}
\frac{1}{2} M^{2}+\int_{T}^{t} m x^{\prime} f d t+\sum_{i=1}^{k} F_{i} \int_{0}^{x} g_{i}(x) d x \\
=c^{2}+\sum_{i=1}^{k} \int_{T}^{t}\left(\int_{0}^{x} g_{i}(x) d x\right) d F_{i} .
\end{aligned}
$$

To prove that Theorem 2 holds for the equation (5.6), we replace $X_{2 n+1}^{2 i} / 2 i$ by the integral

$$
\int_{0}^{x_{2 n+1}} x^{n(i)} u_{i}(x) d x
$$

in the proof of Theorem 2 .

\section{REFERENCES}

1. W. F. Osgood, On a theorem of oscillation, Bull. Amer. Math. Soc. vol. 25 (1919) pp. 216-221.

2. C. T. Taam, The boundedness of the solutions of a differential equation in the complex domain, Pacific Journal of Mathematics vol. 2 (1952) pp. 643-654.

3. Z. Butlewski, Sur les intégrales d'une équation différentielle du second ordre, Mathematica (Cluj) vol. 12 (1936) pp. 36-48.

4. E. J. McShane, Integration, Princeton, 1947.

5. W. Leighton, Bounds for the solutions of a second order linear differential equation, Proc. Nat. Acad. Sci. U.S.A. vol. 35 (1949) pp. 190-191.

6. E. L. Ince, Ordinary differential equations, New York, 1944.

The Catholic University of America 